Homology, Homotopy and Applications, vol.6(1), 2004, pp.1-3

\title{
THE 3-BY-3 LEMMA FOR REGULAR GOURSAT CATEGORIES
}

\author{
STEPHEN LACK \\ (communicated by George Janelidze)
}

\section{Abstract}

We prove a $3 \times 3$ lemma for regular Goursat categories.

The classical " $3 \times 3$ lemma" in abelian category theory involves short exact sequences. In more general contexts - in particular in a category which is not pointed - it makes sense to replace short exact sequences by "exact forks": these are diagrams

$$
R \underset{r_{2}}{\stackrel{r_{1}}{\longrightarrow}} A \stackrel{r}{\longrightarrow} B
$$

in which $\left(r_{1}, r_{2}\right)$ is the kernel pair of $r$, and $r$ is the coequalizer of $r_{1}$ and $r_{2}$. The corresponding $3 \times 3$ lemma would then concern a diagram

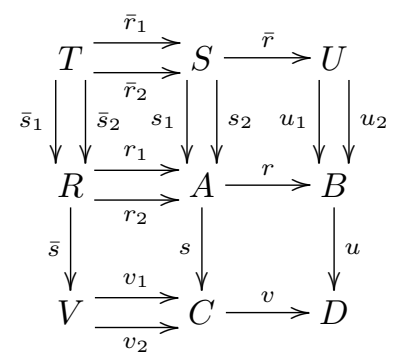

satisfying the usual commutativity conditions (such as $s_{i} \bar{r}_{j}=r_{j} \bar{s}_{i}$ ) in which the three columns and the middle row are exact. The lemma states that the top row is exact if and only if the bottom row is exact, and was proved in [1] to hold in any a regular Mal'cev category. If one is not interested in any of the side issues discussed in [1], a very short proof is possible, in the slightly more general context of a regular Goursat category. The method of proof is due to Lambek: see $[\mathbf{3}]$ and the references therein.

Recall that in any regular category $\mathbb{C}$ one can define the category $\operatorname{Rel}(\mathbb{C})$ of relations in $\mathbb{C}$, in which the objects are the objects of $\mathbb{C}$, but the morphisms are the relations. A regular category is Mal'cev if and only if the equation $R S=S R$ holds for any relations $R: A \rightarrow A$ and $S: A \rightarrow A$ on the same object $A$; the regular category is Goursat when the weaker equation $R S R=S R S$ holds. See $[\mathbf{2}]$ for more information on Mal'cev and Goursat categories.

Received December 17, 2003, revised December 19, 2003; published on February 6, 2004 2000 Mathematics Subject Classification: 18G50, 18A32, 18B10.

Key words and phrases: Non-abelian homological algebra, calculus of relations, Mal'cev category. (C) 2004, Stephen Lack. Permission to copy for private use granted. 


\section{Proof of the lemma}

Under no assumptions on the category at all, a straightforward diagram chase gives:

(a) if $\bar{r}$ is epi then $v$ is the coequalizer of $v_{1}$ and $v_{2}$, and

(b) if the pair $\left(v_{1}, v_{2}\right)$ is jointly monic then $\left(\bar{r}_{1}, \bar{r}_{2}\right)$ is the kernel pair of $\bar{r}$.

In particular, the fact that the bottom row is a coequalizer if the top one is so is very well known. It remains to show:

(c) if the bottom row is exact then $\bar{r}$ is regular epi, and

(d) if the top row is exact then $\left(v_{1}, v_{2}\right)$ is the kernel pair of $v$.

Suppose that the category $\mathbb{C}$ is regular Goursat, and work now in $\operatorname{Rel}(\mathbb{C})$. Identify an arrow $f: A \rightarrow B$ in $\mathbb{C}$ with the corresponding relation from $A$ to $B$, and write $f^{*}$ for its opposite. Recall that $f$ is regular epi if and only if $f f^{*}=1$, and that a jointly monic pair $f_{1}, f_{2}: R \rightarrow A$ is the kernel pair of $f$ if and only if $f^{*} f=f_{2} f_{1}^{*}$. Observe also that, by the definition of composition of relations, if in

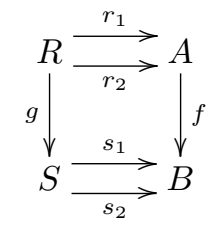

$R$ and $S$ are relations, then $g$ is regular epi if and only if $s_{2} s_{1}^{*}=f r_{2} r_{1}^{*} f^{*}$. If $\left(r_{1}, r_{2}\right)$ and $\left(s_{1}, s_{2}\right)$ are kernel pairs, with coequalizers $r$ and $s$, this is in turn equivalent to $s^{*} s=f r^{*} r f^{*}$.

Then to prove (c) we have:

$$
\begin{aligned}
u^{*} u & =r r^{*} u^{*} u r r^{*} & & \\
& =r s^{*} v^{*} v s r^{*} & & (u r=v s) \\
& =r s^{*} s r^{*} r s^{*} s r^{*} & & \left(v^{*} v=s r^{*} r s^{*} \text { since } \bar{s} \text { is regular epi }\right) \\
& =r r^{*} r s^{*} s r^{*} r r^{*} & & \text { (Goursat }) \\
& =r s^{*} s r^{*} & &
\end{aligned}
$$

and so $\bar{r}$ is indeed regular epi.

To prove $(\mathrm{d})$ :

$$
\begin{array}{rlrl}
v^{*} v & =s s^{*} v^{*} v s s^{*} & & \\
& =s r^{*} u^{*} u r s^{*} & & (u r=v s) \\
& =s r^{*} r s^{*} s r^{*} r s^{*} & & \left(u^{*} u=r s^{*} s r^{*} \text { since } \bar{r} \text { is regular epi }\right) \\
& =s s^{*} s r^{*} r s^{*} s s^{*} & & (\text { Goursat }) \\
& =s r^{*} r s^{*} & \\
& =s r_{2} r_{1}^{*} s^{*} & \\
& =v_{2} \bar{s} \bar{s}^{*} v_{1}^{*} & \left(s r_{i}=v_{i} \bar{s}\right) \\
& =v_{2} v_{1}^{*} &
\end{array}
$$


and so the kernel pair $\left(v_{1}^{\prime}, v_{2}^{\prime}\right)$ will satisfy $v_{i}^{\prime} e=v_{i}$ for some regular epimorphism $e$; we must prove that $e$ is invertible. In the 3 -by-3 diagram now replace $\left(v_{1}, v_{2}\right)$ by $\left(v_{1}^{\prime}, v_{2}^{\prime}\right)$, and replace $s$ by es. Then the three rows, the central column and the right column are all exact, so by (a) and (c) the left column is exact. Thus $s$ and es are both coequalizers of $s_{1}$ and $s_{2}$, so $e$ is invertible, and the proof is complete.

\section{References}

[1] D. Bourn, The denormalized $3 \times 3$ lemma, J. Pure Appl. Alg. 177:113-129, 2003.

[2] A. Carboni, G.M. Kelly, and M.C. Pedicchio, Some remarks on Maltsev and Goursat categories, Appl. Cat. Struct. 1:385-421, 1993.

[3] A. Carboni, J. Lambek, and M.C. Pedicchio, Diagram chasing in Mal'cev categories, J. Pure Appl. Alg. 69:271-284, 1991.

This article may be accessed via WWW at http://www.rmi.acnet.ge/hha/ or by anonymous ftp at

ftp://ftp.rmi.acnet.ge/pub/hha/volumes/2004/n1a1/v6n1a1.(dvi,ps,pdf)

Stephen Lack s.lack@uws.edu.au

School of Quantitative Methods and Mathematical Sciences

University of Western Sydney

Locked Bag 1797 Penrith South DC NSW 1797

Australia 